\title{
Contribution of Lymphatic Absorption to Loss of Ultrafiltration and Solute Clearances in Continuous Ambulatory Peritoneal Dialysis
}

\author{
Robert A. Mactier, Ramesh Khanna, Zbylut Twardowski, Harold Moore, and Karl D. Nolph \\ Division of Nephrology, Department of Medicine, University of Missouri Health Sciences Center, Harry S. Truman Veterans \\ Administration Hospital and Dalton Research Center, Columbia, Missouri 65212
}

\begin{abstract}
The contribution of peritoneal cavity lymphatic absorption to ultrafiltration kinetics and solute clearances in continuous ambulatory peritoneal dialysis was evaluated in patients with normal (group 1) and high (group 2) peritoneal permeability $X$ area during 4-h exchanges using 2 liters $2.5 \%$ dextrose dialysis solution with $30 \mathrm{~g}$ added albumin. Cumulative lymphatic drainage in all continuous ambulatory peritoneal dialysis (CAPD) patients averaged $358 \pm 47 \mathrm{ml}$ per 4-h exchange and reduced cumulative net transcapillary ultrafiltration at the end of the exchange by $58 \pm 7.2 \%$. The peak ultrafiltration volume was observed before osmotic equilibrium between serum and dialysate was reached and occurred when the net transcapillary ultrafiltration rate had decreased to equal the lymphatic absorption rate. Thereafter the lymphatic absorption rate exceeded the net transcapillary ultrafiltration rate, and intraperitoneal volume decreased. Extrapolated to $4 \times 2$ liters, $2.5 \%$ dextrose, 6-h exchanges per d, lymphatic drainage reduced potential daily net ultrafiltration by $83.2 \pm 10.2 \%$, daily urea clearance by $16.9 \pm 1.9 \%$, and daily creatinine clearance by $16.5 \pm 1.9 \%$. Although lymphatic absorption did not differ between the two groups, lymphatic drainage caused a proportionately greater reduction in net ultrafiltration in group $2(P$ $<0.025$ ), because these patients had more rapid dialysate glucose absorption $(P<0.05)$ and less cumulative transcapillary ultrafiltration $(P<0.01)$. These findings indicate that cumulative lymphatic drainage significantly reduces net ultrafiltration and solute clearances in CAPD and that ultrafiltration failure in CAPD occurs when daily lymphatic absorption equals or exceeds daily transcapillary ultrafiltration. Reduction of lymphatic absorption may provide a means for future improvement in the efficiency of CAPD.
\end{abstract}

\section{Introduction}

In continuous ambulatory peritoneal dialysis (CAPD) ${ }^{1}$ net removal of fluid (net ultrafiltration) is induced by osmotic pres-

This work was presented in part at the Annual Meeting of the American Society of Nephrology at Washington, DC on 7 December 1986 and published in abstract form (1987. Kidney Int. 31:252).

Address correspondence and reprint requests to Ramesh Khanna, M.D., Associate Professor of Medicine, MA436 Nephrology Division, University of Missouri Health Sciences Center, Columbia, MO 65212.

Received for publication 14 October 1986 and in revised form 17 June 1987.

1. Abbreviations used in this paper: CAPD, continuous ambulatory peritoneal dialysis; UF, ultrafiltration.

J. Clin. Invest.

(c) The American Society for Clinical Investigation, Inc.

$0021-9738 / 87 / 11 / 1311 / 06 \$ 2.00$

Volume 80, November 1987, 1311-1316 sure (1), and clearances of low-molecular-weight solutes are directly related to the drain volume (2). The end lymphatics of the peritoneal cavity, located mainly on the undersurface of the diaphragm $(3,4)$, continuously drain intraperitoneal fluid by bulk transport (5) and return the absorbed fluid to the venous circulation via the right lymph duct (70-80\%) and thoracic duct (20-30\%) (6). Thus, assuming intraperitoneal residual volume remains constant, the net ultrafiltration (UF) volume at the end of an exchange equals cumulative net transcapillary water transport minus lymphatic absorption during the exchange, and drain volume equals infusion volume of dialysis solution plus cumulative net transcapillary UF minus lymphatic drainage. Lymphatic absorption in hepatic ascites may exceed 10 liters/d $(7,8)$ and in malignant ascites is $>1.2$ liters/d unless there is tumor invasion of the diaphragmatic or mediastinal lymphatics (9). The large intraperitoneal fluid volumes in CAPD should also increase intraperitoneal hydrostatic pressure (10) and ensure constant fluid contact with the subdiaphragmatic peritoneum. Hence, it would not be unexpected if lymphatic drainage in CAPD also exceeds the 1 liter/ d reported in normal man (4) and so significantly reduces net UF and drain volumes.

This study was performed to evaluate the role of peritoneal cavity lymphatic absorption in UF kinetics and loss of solute clearances in CAPD.

\section{Methods}

Subjects. We studied 10 CAPD patients (six men and four women). Their mean age was $58 \mathrm{yr}$ (range, 31-73), and mean duration of CAPD was 35 mo (range, 1-105). Although six of the subjects had had 16 previous episodes of peritonitis, no patient had peritonitis within 3 mo of study. The study was approved by the Institutional Review Board for Human Experimentation, University of Missouri Health Sciences Center, and each patient gave informed consent.

Two groups of patients were identified for analysis of results. Using effluent dialysate/serum creatinine and effluent/initial dialysate glucose ratios during 4- $\mathrm{h}$ exchanges as indices of peritoneal permeability $X$ area, six patients (group 1) had permeability $X$ area within 1 SD and four (group 2) had permeability $X$ area $>1$ SD above the mean of our CAPD population. That is, group 2 had high creatinine and low glucose ratios at any time compared with the CAPD population as a whole.

Modified equilibration test. Each study was performed after an overnight exchange (10-12 h) using 2 liters $2.5 \%$ dextrose dialysis solution. After complete drainage of the overnight dwell over $20 \mathrm{~min}, 2$ liters $2.5 \%$ dextrose dialysis solution with $30 \mathrm{~g}$ added albumin $(25 \%$ human serum albumin) was infused over $10 \mathrm{~min}$. During infusion the patient rolled from side to side every 2 min to promote intraperitoneal mixing of dialysate. After complete infusion (time 0 ) the patient remained supine for the 4-h study. After $4 \mathrm{~h}$ the dialysate was drained over $20 \mathrm{~min}$, drain volume measured, and 2 liters dialysis solution without albumin instilled. Dialysate samples $(10 \mathrm{ml})$ were obtained serially at times $0,30,60,120$, and 180 min during the study dwell, from each infusion and drain volume, and immediately after complete infusion of the new bag after the study dwell. Serial samples were taken 
after draining $200 \mathrm{ml}$ into the drain bag and inverting the bag three times. After each serial sample was obtained, dialysate remaining in the drain bag was immediately reinfused. Blood samples $(10 \mathrm{ml})$ were drawn immediately pre- and poststudy.

Repeat equilibration studies were performed in four patients within the next $4 \mathrm{~d}$. Three of these patients were from group 1 and one from group 2 . The above protocol was followed using 2 liters Ringer's lactate with $25 \mathrm{~g}$ albumin instead of 2 liters $2.5 \%$ dextrose, $1.5 \%$ albumin solution and $23.4 \% \mathrm{NaCl}$ was added to the Ringer's lactate solution ( $273 \mathrm{mOsm} /$ liter) to correct the dialysate osmolality to each patient's measured serum osmolality. These studies enabled comparison of lymphatic absorption rates during 4-h exchanges with equal volumes of isotonic and hypertonic intraperitoneal fluid. A third 2-h study using 2 liters $2.5 \%$ dextrose dialysis solution was performed in these four patients to compare peritoneal permeability $X$ area with and without added albumin with calculated and measured 2-h intraperitoneal volumes.

Laboratory methods. Dialysate and serum urea and creatinine and serum glucose were measured by standard automated methods. Dialysate creatinine values were corrected for high-dialysate glucose concentrations (11). Dialysate and serum albumin concentrations were measured by the bromcresol green method (12) and dialysate glucose by an orthotoluidine method (13). Serum and dialysate osmolality were determined using a $5100 \mathrm{~B}$ vapor pressure osmometer (Wescor Inc., Logan, UT).

Calculations. Because peritoneal cavity lymphatics drain intraperitoneal fluid by bulk transport without increase or decrease in protein content $(14,15)$ and intraperitoneal macromolecules of mol wt $>20,000$ are returned to the venous circulation almost exclusively by the peritoneal lymphatics $(16,17)$, lymphatic absorption in CAPD can be calculated from the rate of disappearance of albumin from the peritoneal cavity. Net transcapillary UF was estimated from the dilution of the initial dialysate albumin concentration because the intraperitoneal albumin concentration is unchanged by lymphatic absorption of intraperitoneal fluid and any decrease in the dialysate albumin concentration results from the net influx of fluid from the pertioneal microcirculation. That is, $(a)$ net transcapillary UF $(\mathrm{ml})$ at time $t(\mathrm{~h})$ $=\mathrm{C}_{o} / \mathrm{C}_{t}\left(\mathrm{IPV}_{o}\right)-\left(\mathrm{IPV}_{o}\right) ;(b)$ lymphatic absorption during the 4-h dwell $(\mathrm{ml})=\mathrm{C}_{\mathrm{o}} / \mathrm{C}_{\mathrm{G}}\left(\mathrm{IPV}_{o}\right)-\mathrm{C}_{4} / \mathrm{C}_{\mathrm{G}}\left(\mathrm{IPV}_{4}\right) ;(c)$ calculated net UF at $4 \mathrm{~h}(\mathrm{ml})$ $=$ net transcapillary UF at $4 \mathrm{~h}$ - lymphatic absorption during the 4-h dwell; and $(d)$ measured net UF at $4 \mathrm{~h}(\mathrm{ml})=\left(\mathrm{IPV}_{4}\right)-\left(\mathrm{IPV}_{o}\right)$, where $\mathrm{C}_{o}, \mathrm{C}_{4}, \mathrm{C}_{t}=$ dialysate albumin concentration at 0,4 , and $t \mathrm{~h}$ dwell time, respectively, $\mathrm{C}_{\mathrm{G}}=$ geometric mean dialysate albumin concentration $=\sqrt{\left(\mathrm{C}_{0} \times \mathrm{C}_{4}\right)}, \mathrm{IPV}_{o}=$ intraperitoneal vol at $0 \mathrm{~h}$ dwell time $=$ infusion vol + preequilibration residual vol, and $\mathrm{IPV}_{4}=$ intraperitoneal vol at $4 \mathrm{~h}$ dwell time $=$ drain vol + sample vol + postequilibration residual vol.

Thus, net transcapillary UF is defined as the net value of bidirectional transcapillary water movement into the peritoneal cavity during a time interval. Net UF (calculated and measured) represents the increase in intraperitoneal volume over a time interval.

The equation for calculating net transcapillary UF relies on the fact that most of the ultrafiltration occurs early in the exchange (1) and dilutes the dialysate albumin concentration before the intraperitoneal albumin pool is significantly reduced by absorption by lymphatics. Later in the dwell time net transcapillary UF approaches zero and albumin losses via convective flow of fluid into lymphatics proceed with little or no change in dialysate albumin concentration. Consequently the dialysate albumin concentration decreases almost exponentially during the exchange due to ultrafiltration. The geometric mean $\left(C_{G}\right)$ closely represented the time-averaged intraperitoneal albumin concentration and allowed calculation of lymphatic absorption with two data points. Pre- and postequilibration residual volumes were derived from the mean of each residual volume calculated from the change induced in the drain dialysate urea, creatinine, glucose, and albumin concentrations by the known new infusion volume.

Daily transcapillary UF and drain volume with $4 \times 2$ liters, $2.5 \%$ dextrose dialysis solution were extrapolated by assuming transcapillary UF was complete after $4 \mathrm{~h}$ and lymphatic absorption was continuous at the observed rate. Daily solute clearances $=($ drain volume per $d$ $X$ drain dialysate solute concentration)/serum solute concentration, where the drain dialysate concentration was assumed to equal the 4-h solute concentration. Reverse solute clearances (via lymphatics) were similarly calculated based on the mean dialysate solute concentration per exchange. Thus reverse solute clearances $=$ (lymphatic drainage per $\mathbf{d} \times$ mean dialysate solute concentration)/serum solute concentration.

Results of groups 1 and 2 were compared by Student's $t$ test.

\section{Results}

Mean peritoneal cavity lymphatic absorption during the 4-h dwell in the 10 CAPD patients was $358 \pm 47$ (SEM) $\mathrm{ml}$ (Table I). Cumulative lymphatic absorption during the 4-h exchange reduced the cumulative net transcapillary UF volume at $4 \mathrm{~h}$ by $58 \pm 7.2 \%$ (Table I, Fig. 1). Calculated and directly measured net UF volumes at $4 \mathrm{~h}$ were closely related $(r=0.96)$, supporting the validity of the methods (Table I).

The peak net UF volume was observed before the $2-\mathrm{h}$ dwell time (Fig. 1) and represented the exchange time when the rate of lymphatic absorption equalled the rate of net transcapillary UF (Fig. 2). After the peak UF volume, and thus the maximum intraperitoneal volume, lymphatic flow rate exceeded the net transcapillary UF rate (Figs. 1 and 2). Net transcapillary UF rate decreased exponentially to near 0 at $4 \mathrm{~h}$ (Fig. 2). Peak intraperitoneal volume preceded osmotic equilibrium which in turn preceded glucose equilibrium (Fig. 3).

During the studied exchanges effluent/serum creatinine and effluent/initial dialysate glucose ratios of groups 1 and 2 were significantly different $(P<0.02$ and $P<0.05$, respectively) but correlated with solute ratios during equilibration studies without albumin performed in the preceding year ( $r$ $=0.83$ ) (Fig. 4). Although lymphatic absorption did not differ

Table I. Ultrafiltration Kinetics during 4-h Exchanges Using 2 Liters 2.5\% Dextrose Dialysis Solution

\begin{tabular}{|c|c|c|c|c|}
\hline & $\begin{array}{l}\text { Groups } 1 \\
\text { and } 2\end{array}$ & Group 1 & Group 2 & $P^{*}$ \\
\hline Transcapillary UF $(m l)$ & $669 \pm 90$ & $834 \pm 92$ & $421 \pm 82$ & $<0.01$ \\
\hline Lymphatic absorption $(m l)$ & $358 \pm 47$ & $399 \pm 76$ & $296 \pm 24$ & NS \\
\hline Calculated net UF $(\mathrm{ml})$ & $310 \pm 67$ & $435 \pm 65$ & $125 \pm 63$ & $<0.005$ \\
\hline Measured net UF $(\mathrm{ml})$ & $321 \pm 69$ & $460 \pm 58$ & $114 \pm 60$ & $<0.005$ \\
\hline Lymphatic absorption/transcapillary UF (\%) & $58 \pm 7.2$ & $45 \pm 6.8$ & $76 \pm 8.8$ & $<0.025$ \\
\hline
\end{tabular}

* Mean \pm SEM of groups 1 and 2 were compared by Student's $t$ test. 


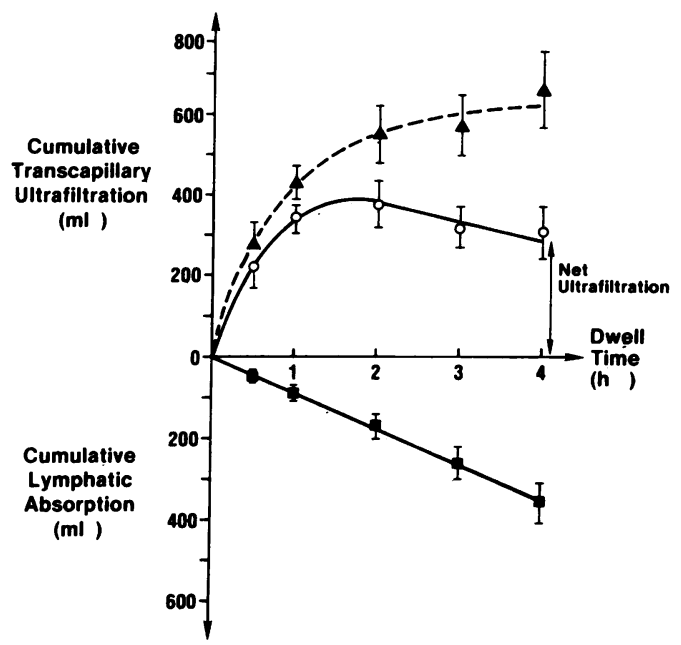

Figure 1. Cumulative lymphatic absorption, net ultrafiltration, and cumulative transcapillary UF (mean \pm SEM) during 4-h exchanges using 2 liters $2.5 \%$ dextrose dialysis solution $(n=10)$.

between the two groups, lymphatic absorption caused a proportionately greater reduction in net UF at $4 \mathrm{~h}$ dwell time in group $2(P<0.025)$ because these patients had less cumulative net transcapillary UF $(P<0.01)$ (Table I, Fig. 5). There was no correlation in our patients between the number of previous episodes of peritonitis and the lymphatic absorption rate.

Extrapolated to $4 \times 2$ liters, $2.5 \%$ dextrose 6 -h exchanges per $d$, daily lymphatic drainage in the 10 patients was $2.15 \pm 0.29$ liters and daily drain volumes $9.0 \pm 0.33$ liters. Thus, lymphatic absorption reduced potential daily net UF volume by $83.2 \pm 10.2 \%$ (Fig. 6) and potential drain volume by $19.1 \pm 2.2 \%$ (Fig. 7). Daily lymphatic (reverse) urea and creatinine clearances were $1.8 \pm 0.23$ liters and $1.49 \pm 0.18$ liters, respectively, reducing potential daily urea and creatinine clearances by $16.9 \pm 1.9 \%$ and $16.5 \pm 1.9 \%$, respectively (Fig. 7). Daily solute clearances between groups 1 and 2 did not differ but daily net UF was significantly lower in group $2(P<0.005)$ (Fig. 6).

Net fluid absorption during the $4 \mathrm{~h}$ after intraperitoneal instillation of 2 liters, near isosmotic fluid in four of the pa-

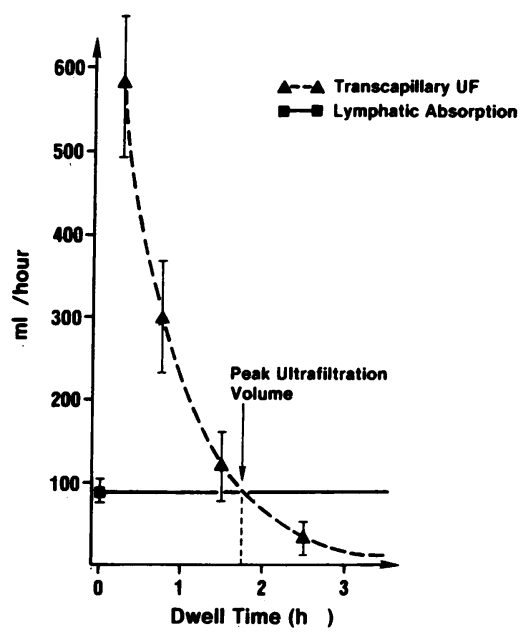

Figure 2. Transcapillary UF and lymphatic absorption rates during exchanges using 2 liters $2.5 \%$ dextrose dialysis solution. Peak ultrafiltration volume (arrows) occurs when transcapillary UF rate equals lymphatic flow rate.
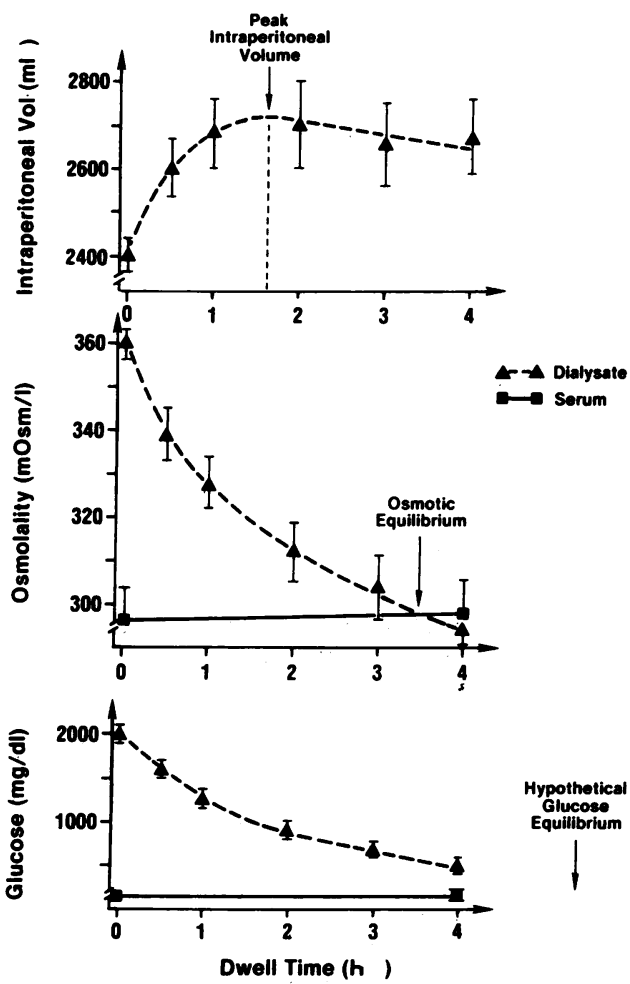

Figure 3. Intraperitoneal volume, serum and dialysate osmolality, and glucose (mean $\pm \mathrm{SEM}$ ) during 4-h exchanges using 2 liters $2.5 \%$ dextrose dialysis solution $(n=10)$. Arrows indicate peak intraperitoneal volume, osmotic equilibrium, and hypothetical glucose equilibrium.

tients was $255 \pm 38 \mathrm{ml}$ (Fig. 8). Reduction in intraperitoneal volume was observed without significant change in the concentration of albumin added to the dialysate (Fig. 8). In these four patients cumulative lymphatic absorption over $4 \mathrm{~h}$, calculated from the rate of removal of intraperitoneal albumin, was $343 \pm 167 \mathrm{ml}$ with Ringer's lactate and $333 \pm 87 \mathrm{ml}$ with $2.5 \%$ dextrose peritoneal dialysis exchanges.

In the same four patients, directly measured intraperitoneal volumes $2 \mathrm{~h}$ after infusion of 2 liters $2.5 \%$ dextrose without albumin were $2,604 \pm 51 \mathrm{ml}$, and calculated 2 -h intraperitoneal volumes with $2.5 \%$ dextrose with added albumin were $2,663 \pm 154 \mathrm{ml}(r=0.98)$. Dialysate serum solute concentration ratios at $2 \mathrm{~h}$ during studies with $2.5 \%$ dextrose without albumin in these four patients correlated $(r=0.75)$ with dialy-

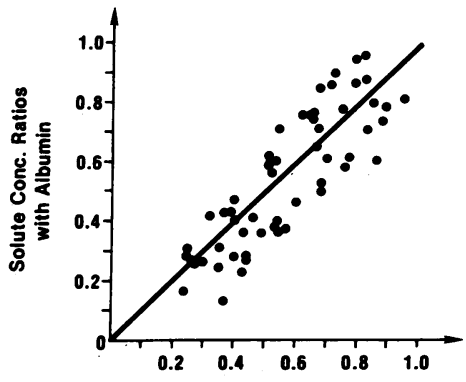

Solute Conc. Rątios without Albumin
Figure 4. Dialysate and serum creatinine and urea and effluent initial dialysate glucose ratios during exchanges using 2 liters $2.5 \%$ dextrose dialysis solution with added albumin correlated with solute ratios during equilibration studies using 2 liters $2.5 \%$ dextrose dialysis solution without albumin performed in the preceding year. 


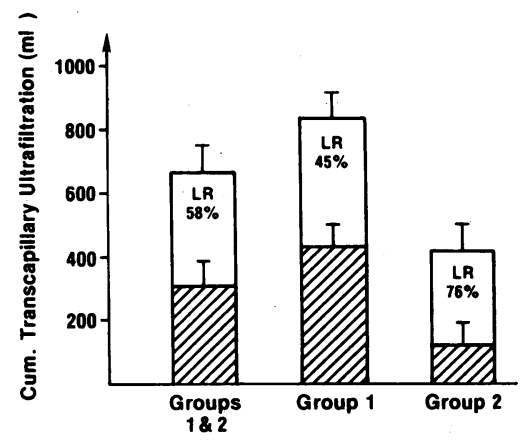

LA Cumulative Lymphatic Drainage during a $\mathrm{h}$ Det Uitrafiltration Volume at $4 \mathrm{~h}$
Figure 5. Cumulative transcapillary UF, lymphatic drainage, and net $\mathrm{UF}($ mean \pm SEM) at $4 \mathrm{~h}$ dwell time using 2 liters $2.5 \%$ dextrose dialysis solution in CAPD patients with normal (group 1) and high (group 2) peritoneal permeability $\times$ area.

sate serum solute ratios at $2 \mathrm{~h}$ with $2.5 \%$ dextrose and Ringer's lactate with added albumin (Fig. 9). These results and Fig. 4 indicate that addition of $30 \mathrm{~g}$ albumin to $2.5 \%$ dextrose dialysis solution did not significantly change peritoneal permeability $X$ area or cumulative transcapillary UF.

\section{Discussion}

This study demonstrates that cumulative peritoneal cavity lymphatic absorption significantly reduces net UF and solute clearances in CAPD patients. The peritoneal lymphatic absorption rate in our CAPD patients was $89.5 \pm 11.8 \mathrm{ml} / \mathrm{h}$, which is consistent with prior observations in patients with hepatic $(9,13)$ and malignant $(9,18)$ ascites who have no disease of the diaphragmatic or mediastinal lymphatics. Conversely, tumor invasion $(18,19)$ or fibrosis $(20)$ of the subdiaphragmatic lymphatics reduces peritoneal lymphatic drainage and may induce ascites formation.

This considerable rate of lymphatic drainage in CAPD patients has a major role in ultrafiltration kinetics. Net transcapillary ultrafiltration defines net fluid movement from the peritoneal microcirculation into the peritoneal cavity primarily in response to osmotic pressure and allows for any concurrent distal capillary or venular fluid reabsorption; net ultrafiltration

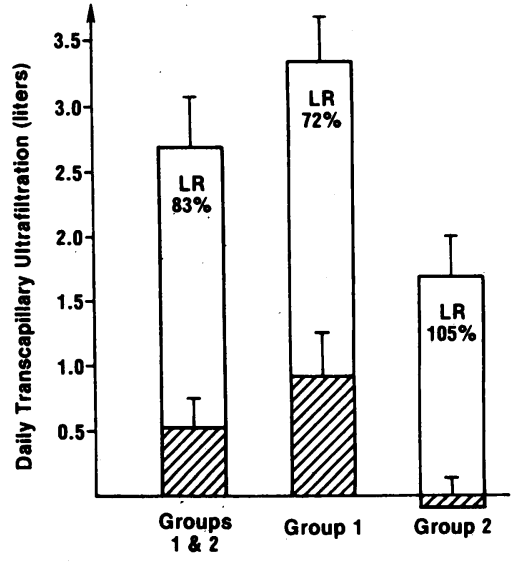

LR Daily Lymphatic Reabsorption Da Daily Not Uitrafiltration
Figure 6. Daily transcapillary UF, lymphatic absorption, and net UF volumes (mean \pm SEM) in groups 1 and 2 using $4 \times 2$ liters $2.5 \%$ dextrose dialysis solution per d.

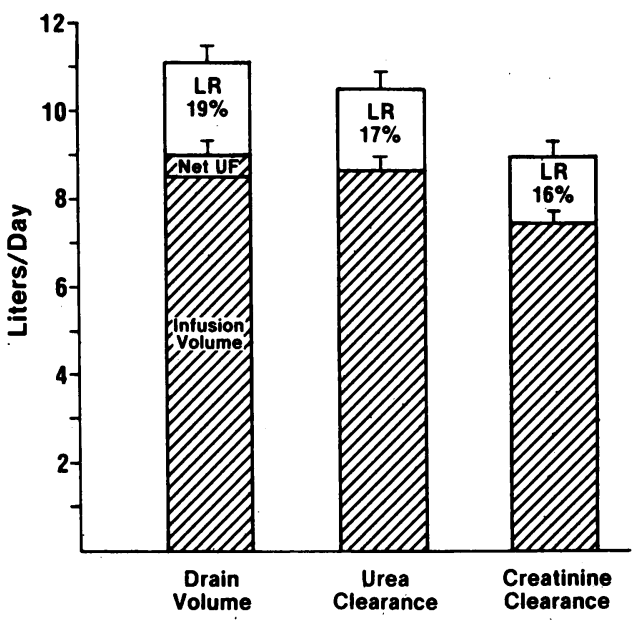

LR Daily Lymphatic Absorption

Daily Observed Drain Volume/Solute Clearance

Figure 7. Loss of potential daily drain volume, urea clearance, and creatinine clearance (mean \pm SEM) due to lymphatic absorption in patients $(n=10)$ using $4 \times 2$ liters $2.5 \%$ dextrose dialysis solution per d.

would equal the resulting increment in intraperitoneal fluid volume if it were not for peritoneal lymphatic absorption. The net transcapillary UF rate decreases exponentially during the dwell time (Fig. 2) due to dissipation of the glucose osmotic gradient secondary to transperitoneal absorption of glucose and dilution of intraperitoneal glucose by the ultrafiltrate. Because net UF equals cumulative net transcapillary UF minus cumulative lymphatic drainage during the exchange, the net UF rate reaches zero while transcapillary UF continues (Figs. 1 and 2) before osmotic equilibrium (Fig. 3). Thereafter the lymphatic absorption rate exceeds the transcapillary UF rate (Fig. 2) and the UF and intraperitoneal volumes decrease. Thus, peak UF and intraperitoneal volumes are observed before osmotic equilibrium when the lymphatic absorption rate equals
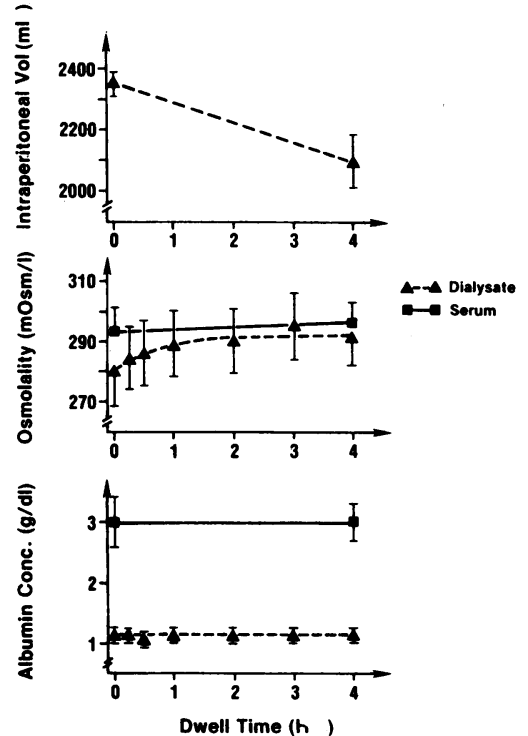

Figure 8. Net fluid absorption, serum and dialysate osmolality, and albumin concentration (mean \pm SEM) during 4-h exchanges using 2 liters, near isosmotic Ringer's lactate with added albumin $(n=4)$. The initial albumin concentration was $<1.2$ $\mathrm{g} / \mathrm{dl}$ due to dilution of the infused solution by the residual volume of the preceding exchange. 


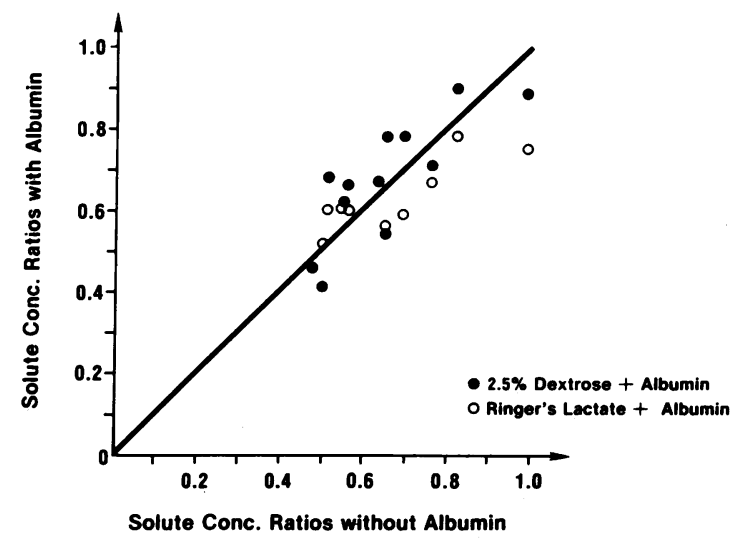

Figure 9. Dialysate/serum urea and creatinine and effluent/initial dialysate glucose ratios during equilibration studies using 2 liters $2.5 \%$ dextrose or Ringer's lactate with albumin correlated with dialysate solute ratios during exchanges using 2 liters $2.5 \%$ dextrose dialysis solution without albumin performed within the next $4 \mathrm{~d}$.

the net transcapillary UF rate and the net UF rate is 0 . Osmotic equilibrium most likely precedes glucose equilibrium because of solute sieving with $\operatorname{UF}(1,21)$ and the higher peritoneal reflection coefficient of glucose than other dialysate solutes (22) may allow net transcapillary UF to continue at a slow rate after osmotic equilibrium (Figs. 1 and 3).

The CAPD patients with high peritoneal permeability $X$ area (group 2 ) had more rapid glucose absorption $(P<0.05)$ and less cumulative transcapillary UF than patients with normal pertioneal permeability $\times$ area (group 1). Thus, although cumulative lymphatic drainage did not differ between the two groups, group 2 had a greater proportion of transcapillary UF reabsorbed by the peritoneal lymphatics and less net UF (Fig. 5). Extrapolated to $4 \times 2$ liters, $2.5 \%$ dextrose dialysis solution exchanges per d, patients in group 2 had negative UF (Fig. 6), even though net transcapillary UF averaged 1.7 liters/d. Therefore, failure of UF in CAPD occurs when daily lymphatic drainage exceeds daily net transcapillary UF. Consequently, because transcapillary UF is observed mainly during the first 2 $h$ of an exchange, whereas lymphatic reabsorption is continuous, net UF can be increased by reducing the exchange time.

Because the lymphatic reabsorption rate was calculated from the rate of disappearance of intraperitoneal albumin, it was important to exclude extraperitoneal dialysate leaks in our CAPD patients. None had clinical evidence of dialysate leakage and three had no evidence of leakage after intraperitoneal infusion of contrast (23). Furthermore two findings in the study suggest that there was no significant adsorption of albumin to the mesothelium of the peritoneal membrane. Firstly, after infusion of $30 \mathrm{~g}$ albumin in the study dwell, the mass transfer of albumin in the drain dialysate was $22.8 \pm 0.8 \mathrm{~g}$, whereas only $1.8 \pm 0.4 \mathrm{~g}$ was removed in the postequilibration in and out exchange. The latter can be fully accounted for by the albumin content of the study residual volume. Secondly, the albumin concentration remained constant during absorption of isotonic intraperitoneal fluid (Fig. 8). Previous studies in a rat model of peritoneal dialysis have shown that directly measured absorption of near isosmotic fluid correlated with calculated fluid absorption by the albumin method $(r=0.98, P$ $<0.01$ ) and confirmed that absorption of intraperitoneal isosmotic fluid is mainly translymphatic (24). The studies with 2 liters $2.5 \%$ dextrose dialysis solution without added albumin (Figs. 4 and 9) showed that the addition of $30 \mathrm{~g}$ albumin had no significant effect on peritoneal permeability $\times$ area or UF. Similarly, the validity of the calculations was verified by the close correlation of directly measured and calculated intraperitoneal volumes at $2 \mathrm{~h}$ and net UF at $4 \mathrm{~h}$. The calculated net fluid absorption rates after peak intraperitoneal volume in our CAPD patients (Figs. 1 and 3) are in accord with previous sequential, direct measurements (25) and infer that fluid absorption in CAPD is mainly lymphatic. The calculated lymphatic absorption rate in this study is much higher than in prior reports $(26,27)$ which determined the rate of lymphatic flow from the rate of mass transfer to radio-labeled tracer from the peritoneal cavity to the blood. This latter method underestimates lymphatic drainage since a significant proportion of the small quantity of radio-labeled colloid administered remains in the subperitoneal interstitium (5) and after systemic absorption the tracer equilibrates out of the blood volume (28). It is possible that lymphatic absorption was higher in our studies than in active CAPD patients because the studies were performed in the supine position and fluid contact with the diaphragm may have been more extensive than in the upright posture. Conversely the increase in intraabdominal pressure with upright posture may increase lymphatic absorption in active CAPD patients (10). Future studies should compare absorption rates in supine and upright positions.

Our results indicate that observed UF with $4 \times 2$ liters, $2.5 \%$ dextrose dialysis solution per $\mathrm{d}$ is only $17 \%$ of cumulative net transcapillary UF and that daily reverse (lymphatic) clearances reduce mass transfer of urea and creatinine by $>16 \%$. Thus, estimates of peritoneal mass transfer based on dialysate drain volume and solute concentrations but neglecting translymphatic transport are erroneously low $(29,30)$. Consequently, net UF and solute clearances in CAPD may potentially be increased if lymphatic drainage were reduced. Although macromolecular osmotic agents, such as polyglucose and gelatins, have less transcapillary absorption and induce more prolonged UF than glucose (31), systemic absorption via the peritoneal lymphatics has so far limited their clinical application. Similarly, any particulate matter or plasticizer that enters the peritoneal cavity with the dialysis solution may also be absorbed by the peritoneal lymphatics.

We conclude that, although the physiological role of the peritoneal lymphatics in improving host defenses and maintaining a small volume of isosmotic fluid in the peritoneal cavity is beneficial, peritoneal cavity lymphatic absorption in CAPD is detrimental. Reduction in lymphatic drainage would greatly alleviate the problem of loss of ultrafiltration observed in some CAPD patients $(32,33)$. Further studies are required to elicit whether net ultrafiltration and solute clearances in CAPD can be augmented by pharmacological manipulation of peritoneal cavity lymphatic absorption.

\section{Acknowledgments}

This work was supported by Nephrology Research Funds and we wish to thank Linda Fitzgerald for preparing the manuscript.

\section{References}

1. Nolph, K. D., F. N. Miller, W. K. Pyle, R. P. Popovich, and M. I. Sorkin. 1981. A hypothesis to explain the characteristics of peritoneal ultrafiltration. Kidney Int. 20:543-548. 
2. Nolph, K. D., R. P. Popovich, A. J. Ghods, and Z. J. Twardowski. 1978. Determinants of low clearances of small solutes during peritoneal dialysis. Kidney Int. 13:117-123.

3. Olin, T., and T. Saldeen. 1964. The lymphatic pathways from the peritoneal cavity: a lymphangiographic study in the rat. Cancer Res. 24:1700-1711.

4. Courtice, F. C., and W. J. Simmonds. 1954. Physiological significance of lymph drainage of the serous cavities and lungs. Physiol. Rev. 34:419-448.

5. Flessner, M. F., R. J. Parker, and S. M. Sieber. 1983. Peritoneal lymphatic uptake of fibrinogen and erythrocytes in the rat. Am. J. Physiol. 244:H89-H96.

6. Courtice, F. C., and A. W. Steinbeck. 1950. The lymphatic drainage of plasma from the peritoneal cavity of the cat. Austral. J. Exp. Biol. Med. Sci. 28:161-169.

7. Dumont, A. E., and J. H. Mulholland. 1960. Flow rate and composition of thoracic duct lymph in patients with cirrhosis. $N$. Engl. J. Med. 263:471-474.

8. Courtice, F. C. 1959. Ascites: the role of the lymphatics in the accumulation of ascitic fluid. Med. J. Aust. 26:945-951.

9. Bronskill, M. J., R. S. Bush, and G. N. Ege. 1977. A quantitative measurement of peritoneal drainage in malignant ascites. Cancer. 40:2375-2380.

10. Zink, J., and C. V. Greenway. 1977. Control of ascites absorption in anaesthetised cats. Effects of intraperitoneal pressure, protein and frusemide diuresis. Gastroenterology. 73:1119-1124.

11. Cook, J. G. H. 1975. Factors influencing the assay of creatinine. Ann. Clin. Biochem. 12:219-232.

12. Pierce Chemical Co. 1978. Measuring albumin concentration with Spec-tur BGG. Methods brochure. Pierce Chemical Co., Rockford, IL.

13. Sigma Chemical Co. 1977. Glucose concentrations by an $O$-toluidine method. Technical bulletin No. 635. Sigma Chemical Co., St. Louis, MO.

14. Henriksen, J. H., N. A. Lassen, H. Parving, and K. Winkler. 1980. Filtration as the main transport mechanism of protein exchange between plasma and the peritoneal cavity in hepatic cirrhosis. Scand. $J$. Clin. Lab. Invest. 40:503-513.

15. Nicoll, P. A., and A. E. Taylor. 1977. Lymph formation and flow. Annu. Rev. Physiol. 39:73-95.

16. Aune, S. 1970. Transperitoneal exchange. IV. The effect of transperitoneal fluid transport on the transfer of solutes. Scand. $J$. Gastroenterol. 5:241-252.

17. Lill, S. R., R. H. Parsons, and I. Buhac. 1979. Permeability of the diaphragm and fluid resorption from the peritoneal cavity in the rat. Gastroenterology. 76:997-1001.

18. Coates, G., R. S. Bush, and N. Aspin. 1973. A study of ascites using lymphoscintigraphy with $99 \mathrm{~m}$ Tc-sulfur colloid. Radiology. 107:577-583.

19. Feldman, G. B., and R. C. Knapp. 1974. Lymphatic drainage of the peritoneal cavity and its significance in ovarian cancer. Am. J. Obstet. Gynecol. 119:991-994.

20. Ismail, A. H., and F. S. Mohamed. 1986. Structural changes of the diaphragmatic peritoneum in patients with schistosomal hepatic fibrosis: its relation to ascites. Lymphology. 19:82-87.

21. Rubin, J., E. Klein, and J. D. Bower. 1982. Investigation of net sieving coefficient of the peritoneal membrane during peritoneal dialysis. American Society for Artificial Internal Organs Journal. 5:9-15.

22. Knochel, J. P. 1969. Formation of peritoneal fluid hypertonicity during dialysis with isotonic glucose solutions. J. Appl. Physiol. 27:233-236.

23. Twardowski, Z. J., R. J. Tully, W. K. Nichols, and S. Sunderrajan. 1984. Computerized tomography in the diagnosis of subcutaneous leak sites during CAPD. Peritoneal Dialysis Bulletin. 4:163-166.

24. Nolph, K. D., R. A. Mactier, R. Khanna, and Z. J. Twardowski. 1987. Ultrafiltration kinetics of peritoneal dialysis in the rat: role of lymphatics. Kidney Int. 31:253A.

25. Twardowski, Z. J., and L. Janicka. 1981. Three exchanges with a 2.5 litre volume for continuous ambulatory peritoneal dialysis. Kidney Int. 20:281-284.

26. Rippe, B., G. Stelin, and J. Ahlmen. 1986. Lymph flow from the peritoneal cavity in CAPD patients. In Frontiers in Peritoneal Dialysis. J. F. Maher and J. F. Winchester, editors. Field, Rich and Associates, Inc., New York. 24-30.

27. Daugirdas, J. F., T. S. Ing, V. C. Gandhi, J. E. Hano, W. T. Chen, and L. Yuan. 1980. Kinetics of peritoneal fluid absorption (from the peritoneal cavity) in patients with chronic renal failure. $J$. Lab. Clin. Med. 95:351-361.

28. Flessner, M. F., R. L. Dedrick, and J. S. Schultz. 1985. Exchange of macromolecules between peritoneal cavity and plasma. Am. J. Physiol. 248:H15-H25.

29. Randerson, D. H., and P. C. Farrell. 1980. Mass transfer properties of the human peritoneum. American Society for Artificial Internal Organs Journal. 3:140-146.

30. Nolph, K. D. 1983. Solute and water transport during peritoneal dialysis. Perspectives in Peritoneal Dialysis. 1:4-8.

31. Twardowski, Z. J., R. Khanna, and K. D. Nolph. 1986. Osmotic agents and ultrafiltration in peritoneal dialysis. Nephron. 42:93-101.

32. Slingeneyer, A., B. Canaud, and C. Mion. 1983. Permanent loss of ultrafiltration capacity of the peritoneum in long-term peritoneal dialysis: an epidemiological study. Nephron. 33:133-138.

33. Wideroe, T. E., L. C. Smeby, S. Mjaaland, K. Dahl, K. J. Berg, and T. W. Aas. 1984. Long-term changes in transperitoneal water transport during continuous ambulatory peritoneal dialysis. Nephron. 38:238-247. 